$\begin{array}{ll} & \text { Etnográfica } \\ \text { etnográfica } & \text { Revista do Centro em Rede de Investigação em }\end{array}$

Antropologia

vol. 25 (3) | 2021

Vol. 25 (3)

\title{
Ethos tribal y simbolismo de la guerra: el caso etnográfico de un grupo de reciente contacto en la Amazonia ecuatoriana
}

Ethos tribal e simbolismo da guerra: o caso etnográfico de um grupo de contacto recente na Amazónia equatoriana

\section{Patricio Trujillo Montalvo y Roberto Narváez Collaguazo}

\section{(2) OpenEdition}

\section{Journals}

Edición electrónica

URL: https://journals.openedition.org/etnografica/10767

DOI: 10.4000/etnografica. 10767

ISSN: 2182-2891

\section{Editor}

Centro em Rede de Investigação em Antropologia

Edición impresa

Fecha de publicación: 1 octubre 2021

Paginación: 751-769

ISSN: 0873-6561

Referencia electrónica

Patricio Trujillo Montalvo y Roberto Narváez Collaguazo, «Ethos tribal y simbolismo de la guerra: el caso etnográfico de un grupo de reciente contacto en la Amazonia ecuatoriana», Etnográfica [En línea], vol. 25 (3) | 2021, Publicado el 29 octubre 2021, consultado el 08 enero 2022. URL: http:// journals.openedition.org/etnografica/10767 ; DOI: https://doi.org/10.4000/etnografica.10767

\section{(c) (1) (8)}

Etnográfica is licensed under a Creative Commons Attribution-NonCommercial 4.0 International License. 


\section{Ethos tribal y simbolismo de la guerra: el caso etnográfico de un grupo de reciente contacto en la Amazonia ecuatoriana}

\section{Patricio Trujillo Montalvo \\ y Roberto Narváez Collaguazo}

Los waorani son un grupo cultural de foresta húmeda tropical que habita en la Amazonia ecuatoriana; su contacto formal con la sociedad nacional del Ecuador se realizó a finales de la década de los 50 del siglo pasado por un grupo religioso estadounidense, provocando desde los imaginarios occidentales un violento proceso de cambio en su cosmovisión y ethos. A pesar de los más de 60 años de contacto, la guerra se mantiene vigente como institución social dentro de los imaginarios simbólicos y los guerreros waorani como referentes de identidad, tradición e historia. La guerra y la venganza expresadas en un característico código guerrero ha sido para los waorani un articulador social, pues otorga prestigio a sus participantes y reconstruye el ethos tribal, reafirma alianzas matrimoniales y relaciones de parentesco, siendo entonces una expresión vigente de la cultura tradicional. Este artículo debate desde la investigación etnográfica como la guerra y venganza se convierten en espacios de reafirmación cultural en un contexto moderno.

PALABRAS CLAVE: guerra, violencia, ethos cultural, culturas amazónicas, tradición cultural, muerte con lanzas.

Ethos tribal e simbolismo da guerra: o caso etnográfico de um grupo de contacto recente na Amazónia equatoriana - Os waorani são um grupo cultural de florestas tropicais húmidas que habitam a Amazónia equatoriana; o seu contacto formal com a sociedade nacional equatoriana foi feito no final da década de 1950 do século passado por um grupo religioso americano, provocando no imaginário ocidental um violento processo de mudança na sua visão de mundo e no seu ethos. Apesar de mais de 60 anos de contacto, a guerra continua vigente como instituição social dentro dos imaginários simbólicos, e os guerreiros waorani como referências de identidade, tradição e história. A guerra e a vingança expressas em um código guerreiro característico têm sido para os waorani articuladores sociais, pois prestigiam os seus participantes e reconstroem o ethos tribal, reafirmam as alianças conjugais e as relações de parentesco, sendo então expressões atuais da cultura tradicional. Este artigo discute a partir de pesquisas etnográficas como a guerra e a vingança se tornam espaços de reafirmação cultural em um contexto moderno. 
PALAVRAS-CHAVE: guerra, violência, ethos cultural, culturas amazónicas, tradição cultural, morte com lanças.

TRUjILlo MONTAlVO, Patricio (patricio.trujillo@fiaam.org) Pontificia Universidad Católica del Ecuador

NARVÁEZ COLLAGUAZO, Roberto (robertonarvaezc@gmail.com) Universidad Andina Simón Bolívar

\section{INTRODUCCIÓN: VENGAR Y MATAR CON LANZAS}

En marzo de 2013, un grupo de guerreros de varios poblados waorani ingresaron al interior de la selva, para ejercer una acción de venganza por el ataque y muerte que sufriera una pareja de ancianos, Ompure Omehuai y su esposa Buganei Caiga, en el poblado de Yarentaro (Narváez Collaguazo 2016). La pareja fue asesinada por lanzas atribuidas a un grupo de guerreros pertenecientes a clanes de pueblos indígenas en aislamiento voluntario (PIAV) conocidos como Taromenani (Narváez Collaguazo 2013c). En respuesta, los hijos mayores de los fallecidos organizaron la acción de venganza, implementando el código guerrero waorani, que exige a los familiares asumir la obligación como miembros de la unidad familiar o nanicabo para realizar la represalia. En la acción de venganza, las relaciones de parentesco, y alrededor de ellas el ejercicio de las alianzas fueron la base de la organización de la correría, donde los guerreros waorani asesinaron a más de 30 miembros de la familia PIAV (Narváez Collaguazo 2013c, 2018a).

Además de los hijos, quienes tenían una obligación moral directa para participar en la venganza del anciano asesinado, fueran los hermanos y parientes masculinos, cuñado y el esposo de la relación poliandria de su madre que iniciaron la correría. El grupo se completó con los yernos de Ompure, a quienes sus hijos exigieron una participación activa, al existir acuerdos tácitos por el intercambio matrimonial, y otros waorani de los poblados, siempre con algún lazo de parentesco de sangre o simbólico, en búsqueda de reafirmar esas alianzas preexistentes o como una oportunidad para acumular prestigio, en este caso a través de una acción tradicional sobre la cual en las últimas tres décadas no había existido un espacio para su ejecución.

"Los hijos de Ompure amenazaron a sus cuñados, indicando que si no participaban de la venganza ellos serían los próximos muertos, y en este punto, se integra el elemento matrilocalidad, por el que los hombres viven en el territorio de la mujer, rodeados de sus familiares y por tanto en desventaja para su propia defensa; así la mejor manera para la supervivencia 
es integrándose plenamente al grupo familiar de la esposa" [Narváez Collaguazo, diario de campo, mayo 2013].

De esta manera, se identifica que entre los waorani la venganza es una reacción social enmarcada en código guerrero que se convierte en un articulador de su cultura tradicional, donde las alianzas se establecen a partir de relaciones de intercambio activas que son, para las sociedades, un contrato social (Mauss 2010; Narváez Collaguazo y Trujillo Montalvo 2020). En este caso, la muerte genera un espacio para activar las alianzas, poniendo en evidencia social los compromisos entre grupos familiares, y por tanto los familiares de los muertos reciben el apoyo manifiesto para cualquier incursión de venganza. En otros escenarios, los familiares de los fallecidos pueden exigir el cumplimiento de esos compromisos. De esta manera el "dar" es una obligación de los grupos familiares cercanos para reafirmar las alianzas existentes. De su parte, los familiares reciben esas manifestaciones de compromiso, que serán retribuidas en un futuro en caso de que los aliados así lo requieran.

A pesar de los años de contacto de los waorani, y de la relación mantenida con el Estado-nación, la guerra como acto social no ha desaparecido ni ha sido extirpada dentro de las prácticas culturales, contrario a lo planteado por Boster, Yost y Peeke (2003), quienes afirman que los waorani encontraron en la paz, impuesta por los misioneros evangélicos del Instituto Lingüístico de Verano (ILV) el fin a las guerras internas entre grupos, y la pacificación de los grupos con la imposición de la figura religiosa del pecado y de la trascendencia de las almas. ${ }^{1}$ Sin embargo, la reiteración de hechos de guerra lleva a cuestionar el planteamiento antes expuesto, ya que en este periodo temporal desde la época del contacto (1960) al presente, la sociedad waorani ha visto un vacío en su reproducción cultural. Así, varios hechos de violencia, en los cuales los waorani han manifestado su ser guerrero, nos hacen reflexionar que para ellos no es extraño participar en un ataque o guerra, porque este hecho reafirma su identidad. El ser waorani reconecta al individuo con el ethos tribal donde la pertenencia al grupo se la manifiesta con acciones que conecten con el duranibai (Ima Omene 2012) o época pasada. ${ }^{2}$ Por ello, para los waorani actuales la reafirmación de hechos de muerte (matanzas) y la exhibición de lanzas recuperadas de ataques es una manera de lucir hacia el resto del grupo el resultado del valor utilizado en ingresar a la selva y todos los hechos de guerra de los cuales

l De acuerdo a Boster, Yost y Peeke (2003), el proceso de contacto waorani ejerció un importante cambio en la cultura, principalmente en las acciones guerreras; sin embargo, después de 60 años de contacto, se han dado múltiples eventos de muerte, que rememoran ese pasado y esas tradiciones que no se han perdido aún después de dos generaciones posteriores al contacto y que no vivieron de la forma tradicional waorani.

2 Ima expone el duranibai como la expresión de la forma de vida tradicional waorani, en donde se reproducen los saberes de los ancestros en un vínculo con la selva y el orden social. 
formaron parte, la ritualidad, en fin, la cultura, una exposición para consolidar el prestigio, su reconocimiento social, el ejercicio del ser waorani.

Este artículo es un aporte al entendimiento de la construcción del ethos sobre la guerra de la cultura waorani. Utiliza amplias fuentes etnográficas y etnohistóricas, información recolectada en varias etapas y diferentes espacios geográficos del territorio waorani y del Parque Nacional Yasuni. Explora categorías nativas (Oliveira 1996; Goldman 2003; Soprano 2008) sobre guerra, paz, muerte y venganza que jefes clánicos waorani han desarrollado como una forma-clave para entender desde su testimonio la construcción simbólica del mundo social waorani. Propone que en el ethos tribal waorani existe o se añade un código cultural que sustenta las razones para el asesinato con lanzas o tapaca wente y sobre todo la justificación de la venganza como un derecho a la guerra (Narváez Collaguazo y Trujillo Montalvo 2020). ${ }^{3}$

\section{VENGANZA Y LA BÚSQUEDA DEL RECONOCIMIENTO SOCIAL}

En el evento del 2013, el reconocimiento del hecho, por parte de los atacantes, tenía tras de sí la reivindicación y reafirmación de las prácticas tradicionales relacionadas con la concepción sobre la muerte, el territorio, el orden social y la venganza.

"A mí me dolía lo que murió mi tío, somos hombres, somos guerreros, ahora como ya hicimos ahora estamos en paz [...] Nosotros no fuimos culpables, culpables son Taromenani porque mataron a mi tío, entonces fuimos a atacar a los Taromenani” [entrevista a Orengo Tocari, 2013].

Si bien se consideraba que este tipo de prácticas, de guerra y muerte, habían sido desplazadas por los procesos de cambio cultural en los que estuvieron inmersos los waorani a partir del contacto, este caso expone que el ataque con lanzas o la venganza no "pertenece a la esfera del discurso, al reino de las historias acerca del tiempo antiguo", tal como lo señalaba Rival en 1996 en su estudio sobre la cultura waorani. La autora planteaba que las expresiones que tenían los waorani respecto a la muerte, a finales de los años 80 , la venganza y la guerra habían sido erradicadas por el proceso de aculturación impuesto principalmente por los misioneros evangélicos que realizaron el contacto con los waorani.

"La muerte [para un waorani] siempre es traumática y causa estallidos de ira incontrolables entre los co-residentes que han perdido a un familiar. La agresión que desemboca en la muerte enfurece a los vivos porque significa 
la partida no deseada de un pariente querido... Toda muerte es considerada como resultado de la voluntad humana, siempre es un asesinato. Asesinar es un acto que implica tener control social sobre la vida y la muerte" (Rival 1996: 93).

Durante los años en que los misioneros evangélicos mantuvieron control en el "Protectorado Huaorani", las prácticas de guerra y venganza no desaparecieron en su totalidad. Y en el caso de análisis se evidencia que la cultura sigue aún activa, en el sentido del "ser" waorani - ethos -, de acuerdo con las definiciones tradicionales que existían, esto es cazador, guerrero, defensor de su territorio y de la seguridad de su grupo familiar. En este sentido, una afirmación realizada por Rival sobre la cultura waorani en ese proceso de cambio y de transición señala que "las historias del pasado (duranibai apene) contienen los mensajes culturales que, al ser transmitidos por las generaciones anteriores, tienen que ser obedecidos", y continúa "con excepción de las historias que hablan de este tipo de asesinatos" (Rival 1996: 59), en este caso, esas excepciones no llegaron a ser reales o a establecerse.

La venganza para varios pueblos amazónicos expone la concepción que tienen en torno a la muerte. Así, para los waorani la muerte es concebida como un resultado de la voluntad humana y siempre va a ser un asesinato. La caída de un árbol que afecte a cualquier individuo puede ser vista como la voluntad de alguien por qué ese árbol caiga sobre alguien (shamanismo); la muerte de alguien víctima del ataque con lanzas será relacionada con un atacante; y así con otros hechos. Con lo anterior, matar por venganza es un acto que implica la existencia de un control social sobre la vida y la muerte, como lo señala Rival: "el asesinato por venganza permite a las sociedades retener el control de los procesos de la vida y la muerte” (1996: 59).

La reafirmación de matanza o de haber matado otorga un estatus especial al waorani, un prestigio reconocido por el nanicabo propio y los cercanos, ya que se exhibe su rol y papel como proveedor; en este caso de seguridad para el grupo, al haber eliminado la amenaza de un grupo enemigo con el cual no fue posible establecer alianza alguna. De esta manera, a partir de la práctica de las tradiciones waorani es donde se construye el ethos, o "ideal" de lo que es ser waorani. La acción guerrera permite una conexión del presente con el pasado, de la continuidad cultural waorani, de las nociones culturales relacionadas con el duranibai, donde los waorani tradicionales eran quienes defendían su territorio tradicional y garantizaban la supervivencia del grupo familiar a través de la recolección y cacería, y cuando se requería muerte de los adversarios que constituyen una amenaza a la seguridad del grupo.

"Nosotros decidimos hacer un rato la pena de él [refiriéndose a la muerte de Ompure], nosotros porque es nuestra cultura tenemos que hacer 
enfrentamiento con los Taromenani [...] para mí era bueno, yo ordenaba con mis primos, yo decía, nosotros no somos mujeres, somos hombres, entonces ese rato cogimos una lanza de mi tío finado, entonces llevamos como 17 lanzas, llevamos a la selva" [entrevista a Orengo Tocari, 2013].

La acción de venganza expone la persistencia del ethos tribal waorani, no importa que hayan transcurrido más de 60 años en un proceso intensivo de cambio cultural, en donde muchos elementos materiales e inmateriales externos han llegado a formar parte de la vida cotidiana waorani, pero, a pesar de aquello, aún persiste y consiste en todos aquellos rasgos particulares de una sociedad que la identifican y la cohesionan. Así, la guerra, la venganza de las muertes es un elemento latente en la vida social waorani que construye y reconstruye las formas sociales internas y las de relacionamiento con el exterior, la cual a pesar de no desarrollarse dentro de los parámetros culturales tradicionales, ha generado una adaptación, incorporando elementos externos, y limitándose además de elementos tradicionales, ejerciendo una ritualidad que integra lo tradicional y lo moderno que reafirma la cultura waorani en un nuevo tiempo.

\section{EL RITUAL DE INICIACIÓN}

En el ataque de venganza por la muerte de Ompure, participaron tres ancianos, quienes ya tenían experiencia de guerras contra otros grupos waorani y contra cowuri; sin embargo, en esa época ellos eran jóvenes, posiblemente recién iniciado en este tipo de acciones. Casi 60 años después, ellos fueron los que guiaron al grupo, haciendo conocer a los más jóvenes las estrategias de guerra tradicionales. Así, los familiares de Ompure y Buganei ejecutaron la venganza y el código guerrero, pero incorporando armas de fuego, ejerciendo así un poder mayor sobre el grupo atacado, con una ventaja tecnológica superior, que pudo haber ocasionado muchas más muertes que en un ataque tradicional.

"Ellos mismo, los hijos de Ompure [refiriéndose a Cawime y Tewane], le proponen a Orengo, diciéndole: Orengo, algún rato tu dijiste que eres valiente, ahora si tú quieres, ¿cuál competencia hacemos?" [entrevista a Araba Omehuai, junio 2013].

Del grupo familiar de Ompure, 17 en total, que realizó el acto de guerra, pocos habían participado previamente en algún acto violento o eran niños en la etapa de contacto que para el grupo wepeiri, al que pertenecen, fue finales de la década del 70. Por ello, y a pesar de que las narraciones de guerra y cantos que mantienen la cultura viva, el evento se constituyó en un espacio de iniciación, en un rito a través del cual se pasó desde la carga simbólica componente 
de los cantos y narraciones a un hecho fáctico en el que la experiencia a través de la acción permitió el ejercicio del símbolo, y el paso de la narración al hecho, a la vivencia del símbolo, al símbolo en acción (Narváez Collaguazo y Trujillo Montalvo 2020).

"Los hijos de Ompure fueron quienes lideraron el ataque y organizaron al grupo. Ellos se encargaron de hablar primero con sus cuñados. A Venancio Yeti, esposo de Acape, le dijeron que si él no iba con ellos a vengar a su padre, entonces que ellos le enterrarían con su padre cuando ellos regresaran... Tagae acompañó porque era hermano de Buganei" [entrevista a poblador de Dicaro, junio 2013].

La iniciación es un espacio de transición en el periodo de vida, que permite el paso en diferentes etapas concebidas por las diferentes sociedades, hasta un estadio en el cual se alcanza la plenitud del ejercicio de la cultura. En el caso de los waorani, el estadio es el que consolida al individuo como ser pleno, esto es, con las capacidades para ejercer sus roles y responsabilidades sociales, en el caso de los hombres como un actor eficiente en la relación con el entorno natural donde su accionar garantiza la provisión para la seguridad del grupo familiar; proveedor de alimentos (a partir de la recolección y cacería) o proveedor de seguridad como guerrero.

\section{GUERRA, FRONTERAS Y TERRITORIO}

El proceso de contacto del pueblo waorani se ha caracterizado por formas de violencia sistemática y una relación inequitativa con actores externos a los que denominan cuwuris, sean estos el Estado, misioneros, madereros ilegales, empresas petroleras u otros grupos indígenas amazónicos, dejándolos luego de 50 años de convivencia formal con la sociedad nacional en una condición de marginalidad y con un territorio tradicional fraccionado e invadido (Narváez Collaguazo 2018a; Trujillo Montalvo 201 1; Rivas Toledo y Lara Ponce 2001).

La frontera étnica, vigente entre los waorani y los cuwuris antes del contacto, estuvo marcada por la violencia. La cartografía temprana Carta de la Provincia de Quito (Maldonado 1750) identifica al río Cononaco como Turibuno, término muy similar al utilizado por los waorani, en la actualidad, para nominar a su actual frontera natural del norte, el Turuburu o Doroboro (río Napo), exponiendo así la posible frontera norte de los grupos waorani en movilidad para esos años (Narváez Collaguazo y Trujillo Montalvo 2020). Estas fronteras naturales y denominaciones nos dan nuevas referencias sobre la idea de territorialidad vinculadas a una característica cultural de los waorani: la alta movilidad y el posible origen de varias familias clánicas (Narváez Collaguazo 2018b; Trujillo Montalvo 2018). De hecho, el actual río Shiripuno, en wao 
tiriro, ${ }^{4}$ identificado como Quehueri-ono, ${ }^{5}$ Cuwuri-ono o río de caníbales, designa esa frontera natural que dividía el espacio de vida y subsistencia de aquel donde habitan o se encuentran enemigos naturales o caníbales, ante quienes la única acción cultural válida es la guerra o el ataque en defensa del territorio y del nanicabo. ${ }^{6}$

El "nanicabo" es la palabra para representar al grupo clánico o familiar donde se concentra el orden social waorani, la base de la estructura social y política, que integra a varias familias nucleares alrededor de fogones en una maloca, casa grande u onko. Al interior de cada nanicabo se encuentran relaciones de alianzas por consanguinidad, matrimoniales o simbólicas, relacionadas con hechos shamánicos o guerras contra otros nanicabos, lo que reproduce el imaginario de territorio entre los waorani, sus fronteras y las fronteras con los cuwuri. Los waorani mantienen una complicada relación de parentesco, en donde la poligamia se expresa en dos vías: poliandria, con la relación de una mujer con varios hombres; o la poliginia, un hombre con varias mujeres. Así, las alianzas parentales son fundamentales para el desarrollo y subsistencia del grupo, puesto que de ellas dependía la supervivencia frente a sus enemigos (Beckerman et al. 2009; Macfarlan et al. 2018; Narváez Collaguazo y Trujillo Montalvo 2020; Narváez Collaguazo 2016; Rival 1996; Trujillo Montalvo 201 1; Yost 1989).

El Estado moderno, en su relación con los waorani, mantuvo un interés extractivo, que incidió en la resignificación cultural en sus dinámicas tradicionales de movilidad, lo cual transfiguró a las actuales formas socio organizativas y de conformación de asentamientos y poblados en su territorio (Trujillo Montalvo 2001). A pesar de ello, no se ejercieron cambios en las formas propias de orden social profundo, que se mantienen, a pesar del influjo recibido y el inminente proceso de cambio, como las dinámicas de matrilocalidad y uxorilocalidad, dentro de derechos territoriales de los antepasados de línea materna, hacia donde se movilizan los waorani, en un ejercicio de traslado cíclico (Narváez Collaguazo 2016, 2018a, 2018b; Trujillo Montalvo 2018).

Es pertinente profundizar las concepciones de los waorani respecto al orden social, puesto en muchas sociedades amazónicas, el orden social, las relaciones de parentesco y alianzas tienen como objetivo la defensa del territorio, y no la estructuración de sociedades complejas (Clastres 1981, 2004, 2008); tanto así que la autarquía y la prevalencia de los líderes de cada grupo de familias o nanicabo se consolidaba en las guerras por la defensa del territorio y los medios de subsistencia. De esta manera, con un manejo independiente de cada grupo

4 Lengua del pueblo waorani.

5 En una traducción literal del wao tiriro al castellano, quehueri o cuwuri es caníbal y ono es río, sería río de cuwuris o de caníbales.

6 Nanicabo es el grupo familiar formado por varias unidades domésticas o núcleos familiares emparentados entre sí; nanicaboiri es el plural. 
familiar, en un ejercicio autárquico, el poder no establecía una centralidad, sino este se mantenía en el cuerpo social, que permeaba incluso al espacio femenino, desde el cual se exigía muchas veces la venganza.

La memoria waorani guarda los aspectos vinculados con la cosmovisión, en donde el territorio, la defensa del mismo, la guerra y la muerte con lanzas son parte de su visión de orden y su concepción de mundo. Así mismo, las dinámicas de movilidad se encuentran vinculadas a esta concepción, en donde destaca la figura materna como línea a la cual se retorna en periodos o ciclos, para hacer uso de los recursos dejados por los abuelos.

\section{CÓDIGO GUERRERO WAORANI}

El código guerrero, de acuerdo a este análisis, define el ethos tribal sustentando una particular identidad étnica grupal, siendo parte vital de la construcción de dinámicas y del orden social waorani. Este orden social se articula alrededor del parentesco, y estructura una forma de control al interior de los nanicabo, con diferentes elementos de coerción. Según Trujillo Montalvo (2011, 2016) el código guerrero se desencadena en diferentes circunstancias de orden cultural, vinculadas con su concepción sobre la vida y la muerte, y en donde la pérdida de un pariente es el eje alrededor del cual se generan las vendetas. Así, se activan las dinámicas de guerra bajo las siguientes causas:

- Por asesinato en condiciones que un familiar es atacado y muerto por otros grupos waorani, warani o cowuri, o cuando este no regresa de cacería, lo que implica que fue atacado en la selva.

- Por acciones shamánicas en donde la actuación de un iro (shamán en idioma wao) de otro grupo mata, enferma o genera un mal en cualquier miembro de la familia.

- Por defensa del territorio, cuando extraños ingresan a sus territorios de cacería o recolección o cuando este territorio se ve bajo amenaza por grupos cowuri o waorani enemigos.

\section{EL ETHOS DE LA GUERRA}

Si bien la sociedad waorani era conocida como sociedad no ribereña (Rival 2015), esto no determinaba que no tuvieran conocimientos que les permitieran atravesar ríos o utilizar los recursos de este, y por ello muchas acciones de guerra se daban en zonas hacia donde se dirigía una movilidad cíclica (Narváez Collaguazo 2018b). En estas etapas existen muchos relatos que forman parte de la memoria mítica, y que conservan aún los descendientes y jefes clánicos waorani sobre emblemáticos guerreros wemonte kewenga - Nee anga, Guikita, Iteka, Moipa, Nenkiwi, Minkaye, Enkeri, Awañete, Nenkimo, 
Baiwa, Nampawe, Wepe, Wane, Kemperi, Kemotare, Niwa, quienes defendieron el territorio causando muchas muertes a los cuwuri, entre los ríos: Curaray, Tzapino, Arajuno, Payamino, Villano, Napo (Trujillo Montalvo 2011 ).

Blomberg, en su libro Los Aucas Desnudos (2015), relata con precisión varios hechos que contribuyeron a la creación de la imagen de los feroces "aucas", grupos reacios al contacto y a la civilización. Auca (salvaje desnudo en kichwa), fue la palabra con la que se designó o identificó por mucho tiempo a los clanes waorani que atacaron y defendieron su territorio hasta 1956, fecha en donde se marca una nueva relación con el Occidente, pues se concreta el contacto mediado por el Summer Linguistic Institute-Wycliffe Books Traslators SLI-WBT (Instituto Lingüístico de Verano - ILV), organización evangélica de los Estados Unidos, la misma que había sido contratada por el Estado ecuatoriano para civilizar, pacificar y evangelizar a los aucas, que tanto problema habían causado en la zona de las provincias de Pastaza y Napo (Trujillo Montalvo 2011).

La relectura de innumerables datos etnográficos (escritos de viajeros, misioneros) antes del contacto con el Occidente (Blomberg 2015; Cabodevilla 2010; Gartelmann 1977; Robarchek y Robarchek 1998; Santos Ortiz 1996; Tirdmarsh 1945; Up de Graff 1996; Wallis 1973), indican que este grupo fue catalogado como extremadamente violento, de una altísima movilidad, luchando con cualquier extraño que había ingresado en sus territorios. Esto creó el imaginario de salvajismo, especialmente entre los kichwa quienes los denominaban aucas o salvaje, no civilizado.

Según Cabodevilla (1996, 1999), el territorio entre los ríos Napo y Curaray por la acción cauchera quedó disminuido en su población tradicional de grupos omaguas y zaparas, dando lugar a un espacio territorial vacío que fue ocupado posiblemente por familias waorani, a quienes Yost (1981), en la primera época del contacto con el Instituto Lingüístico de Verano, los identificó en cuatro grupos: Piyemoiri-Nihuairi, Baihuairi, Wepeiri y Guikitairi.

La guerra intra y extra grupal, para los waorani, fue la forma de reproducción cultural del ethos waorani y de otras muchas sociedades amazónicas (Chagnon 1968; Clastres 1981, 2004; Narváez Collaguazo y Trujillo Montalvo 2020; Narváez Collaguazo 2018a; Trujillo Montalvo 2016) que para el caso waorani se integra al orden social tradicional y al orden social contemporáneo. Esto entrelaza complejos sistemas de alianzas y lealtades grupales donde se intercambian formas culturales tanto materiales como simbólicas que en su momento fueron una respuesta o catalizador para resolver sus conflictos clánicos: entre nanicaboiri (mismos clanes), ${ }^{7}$ warani (otros clanes waorani) ${ }^{8}$ y cowuri (extraños, no

7 Entre los waorani es frecuente confirmar la pertenencia a un nanicabo con afirmaciones como: "familia propia", que evidencia la relación de sangre existente con miembros del grupo, que integra también a alianzas a través de matrimonios.

8 Identificados también como waorani, pero sin líneas de parentesco directas o alianzas. Y con quienes se abría la posibilidad de alianza o guerra, dependiendo las condiciones que se pudieran dar. 
waoranis), ${ }^{9}$ en la actualidad persiste como parte del orden social, tanto para consolidar las alianzas producto del matrimonio, o por acuerdos entre nani$c a b o$, y que se encuentran dentro del marco en donde el complejo orden social autárquico persiste y configura los poblados waorani y las relaciones que se establecen al interior de estos (Macfarlan et al. 2018; Narváez Collaguazo y Trujillo Montalvo 2020; Narváez Collaguazo 2016, 2018a, 2018b; Trujillo Montalvo 2011). En su momento, las guerras, o eventos de venganza donde la muerte con lanzas o tapaca wente fuera el principal mecanismo social, reguló la demografía y sobre todo las relaciones simbólicas con sus vecinos étnicos, dando un significado simbólico al código guerrero, convertido dentro de ethos waorani en un derecho cultural.

Si bien las acciones de contacto impulsadas por el Instituto Lingüístico de Verano (ILV) en la década de los 50 del siglo anterior suprimieron varias instituciones sociales y trajeron como resultado un profundo cambio cultural en los waorani, entre ellas los cantos de guerra, no lograron eliminarlos de la memoria, persistiendo y constituyéndose en parte de las tradiciones y de la rememoración e identificación cultural.

"Dorani bay, es la actividad de conversar sobre cómo eran los waodani. A veces se usa leyendas y otras veces cantos o relatos de los(as) abuelos(as) [...] luego de conversar es común que cada uno salga a realizar la actividad que considera, a lo largo de la charla, es su llamado. Por ejemplo, ir a cazar entre hombres o a sacar pescado entre mujeres; seguir cocinando junto con niños y niñas; salir simplemente a conversar con otros parientes; e incluso ir de correría a otras casas más lejanas" (Toca et al. 2009: 59).

Para los waorani la guerra mantiene aún una amplia representación simbólica, en sus relatos, cantos, su memoria oral, su cosmovisión y ethos. Trujillo Montalvo (2011) explica que existen tiempos de paz o de abundancia (waemo eñere) y tiempos de guerra o de escasez (piinte eñere). En las entrevistas realizadas a los guerreros waorani o pikenanis, se evidencia la emoción que sienten al revivir sus experiencias.

Explican que existen dos momentos para definir hechos o etapas de guerra. Una relacionada con eventos de tipo mágico-shamánico, que sería uno de los motivos movilizadores de venganza y muerte con lanzas, puesto involucra muertes de familiares por brujerías enviadas por enemigos.

Muchas de las guerras interclánicas fueron producto de eventos mágicos para eliminar a sus enemigos. La muerte con lanzas al jefe clánico Ompure y su mujer Buganei, ocurrida en marzo del 2013, se debería a que era conocido como Iro o brujo. Al parecer, miembros enfermos de una familia de PIAV 
habrían contactado a Ompure para que los curara. Sin embargo, los enfermos murieron, por lo que miembros de los PIAV relacionaron la muerte de sus familiares a eventos mágicos y brujería hecha por Ompure, y así por venganza lo asesinaron, acusándolo de haber matado a los enfermos (Narváez Collaguazo 2016; Trujillo Montalvo 2016).

En una especie de enfrentamientos o guerras mágicas, shamanes eran muertos en una espiral de violencia que generaba venganzas, odios y sentimientos de revancha por las muertes de familiares. Varios casos de muertes recientes evidencian lo señalado: en 1998 es asesinado un shaman shuar en el río Shiripuno (Cabodevilla 2010), luego en 2000 un shamán kichwa en San José del Curaray, ejemplificando que las muertes shamánicas se siguieron reproduciendo aun cuando uno de los principales preceptos establecidos por los misioneros evangélicos y católicos durante el proceso de contacto era no matar, y para ello una de las reglas era "no hay venganzas" (Boster, Yost y Peeke 2003).

De esta manera, la acción de la venganza por la muerte de los familiares como resultado de prácticas shamánicas se mantuvo después del contacto, en donde estos hechos se dieron de manera puntual y directa contra los shamanes, y no como correrías de guerra, por lo que hay que diferenciar las dinámicas y estrategias implícitas en estos hechos diferenciados.

"En el caso del shamán shuar, la partida guerrera estuvo compuesta por unos cuantos familiares de la víctima. De la misma manera, en la muerte del shamán kichwa los autores fueron el esposo de la víctima muerta por la acción shamánica y dos hermanos menores de edad, exhibiéndose así incluso una ritualidad vinculada con la iniciación. Para el segundo hecho, los atacantes utilizaron pedazos de chonta, no lanzas, y armas de fuego, siendo el fin del ataque la muerte del shamán" [Narváez Collaguazo, diario de campo, 2002].

El ethos tribal de muerte o guerra alrededor de estos actos de asesinatos a shamanes se vinculan con eliminar una amenaza latente, al interior del territorio de vida o fuera de él, que constituye un actor con capacidad del manejo de recursos sobrenaturales que pueden generar la muerte, y por tanto es una amenaza a la sobrevivencia del nanicabo. En ese sentido, el ataque a shamanes representa una acción enmarcada en un contexto de supervivencia, donde se requieren acciones inmediatas, y donde el acto busca eliminar al enemigo con "poder", es decir, un personaje con "valor mágico-religioso" (Moreno Yánez 2018), eliminando así ese poder.

El otro evento es el relacionado con el denominado código guerrero o derecho natural a usar sus lanzas y se basa en la venganza (Trujillo Montalvo $2011)$. 
"La muerte de un familiar de ser vengada como un imperativo, existen miembros de un clan, niños inclusive, que esperan ser adultos para vengar la muerte de sus familiares y poder vivir en paz o tranquilos. [Trujillo Montalvo, diario de campo, 2013]

En época de paz (waemo eñere) la selva como espacio doméstico abre la posibilidad de una serie de relaciones; se establecen alianzas, principalmente matrimoniales, se mantiene una movilidad tradicional relacionada con la estacionalidad, con cacería y recolección de productos de la selva. Además, permite la posibilidad de mantener kewenas, chacras o huertas de productos de periodo corto, como yuca, maní y otros. Por su parte, las épocas de guerra son una manifestación de ruptura de alianzas previamente existentes, o imposibilidad de establecer alianzas potenciales. La movilidad es estratégica, esto es, traslados rápidos por el territorio hacia zonas de los antepasados, con la finalidad de alejarse de los enemigos, o el repliegue hacia el territorio de los antepasados en caso de ejecutar un ataque.

Tradicionalmente los waorani manejaban kewenas, pero su dieta se basaba principalmente en productos de la recolección, que se relacionaba con los periodos de guerra y paz, donde las dinámicas de movilidad variaban. Durante la investigación, Rivas Toledo, en un trabajo etnográfico reciente (2019), identifica la diferencia entre kewenas y kewenkores, en donde los primeros son los cultivos cercanos a los onkos en épocas de paz, mientras los segundos son cultivos abandonados en un marco de guerra, y se vinculan con la noción de selva domesticada, "pues a ellos retornan a buscar recursos en jornadas de huida por la selva o de cacería" [entrevista a Alexis Rivas Toledo, 2019].

Al existir mayor dinamismo, la subsistencia es básicamente de recolección y cacería, teniendo períodos en los que no tienen posibilidad de manejar kewenas sino más bien recurren a los kewenkode, que son las kewenas abandonadas o en desuso por movilidades pasadas. De esta manera, la época de paz se expresa como una época de abundancia, de relaciones activas y dinámicas, mientras que la guerra es una época de escasez, donde la movilidad es más acentuada y donde se imitan las relaciones incluso con los grupos familiares afines.

Para los waorani, la acción individual afecta para bien o mal al nanicabo, por ello las mismas tienen una relación directa con garantizar la sobrevivencia del grupo familiar, mediante la reproducción a través del uso de los recursos de la selva. La recolección es la acción preponderante en la cotidianidad y es la que permite el sustento de un grupo clánico; por ello, la relación con el entorno ambiental se configura sobre la relación con el entorno social inmediato (Narváez Collaguazo 2018b; Rival 2015), y forma fronteras étnicas con aliados y enemigos. Siendo así, la acción colectiva de guerra asegura las condiciones sobre el territorio de vida, en tiempos de paz y en tiempos de guerra (Narváez Collaguazo 2018a). 
Como pueblo de reciente contacto los waorani mantienen muchos elementos culturales tradicionales como las dinámicas de movilidad, la forma de relacionamiento intergrupal, la autarquía y la misma recolección; por su parte, el Estado ha tenido una escasa capacidad para poder plantear una relación equitativa (Trujillo Montalvo 2001).

\section{MUERTE CON LANZAS Y LOS SÍMBOLOS DE LA GUERRA}

Para Manuel "Wane" Cahuia, jefe clánico del poblado waorani de Noneno, ubicado a orillas del río Shiripuno (Quehueri-ono), y vecino de grupos familiares PIAV, la decoración de las lanzas expresa la intención del fabricante y la carga emocional de este para la acción de matar, entrelazando estas acciones con la forma natural de vengar o con el código guerrero (Narváez Collaguazo y Trujillo Montalvo 2020).

"Lanza con plumas negras de gallinazo son para que el cuerpo del muerto se pudra y sea comido por estos animales. Lanza con pelos de oso banderón son para demostrar la fuerza y el coraje que tiene este animal para enfrentar a cualquier enemigo. Lanza con plumas rojas o pintadas con chonta son decoradas de esta manera para que la lanza se llene de sangre. Lanza con plumas de águila arpía demuestran la fuerza del guerrero para atacar" [entrevista a Manuel Cahuia, Octubre 2009, río Shiripuno-Quehueriono].

Tradicionalmente los waorani mantenían un ritual para la elaboración de lanzas, que iniciaba con el establecimiento de un acuerdo con parientes y aliados para la guerra, ya sea para defensa o para ataque, integrando a los individuos de sexo masculino en jornadas de corte de palmas de chonta, o lo que los waorani denominan "tumbar chontas". Tumbar la chonta tiene un significado determinante, ya que la chonta significa la conexión con los antepasados, con los abuelos que sembraron esas chontas, así mismo, son la fuente de un alimento fundamental que fructifica cada año, y por lo que cada nanicabo genera una movilidad estacionaria para acceder a este alimento y también para realizar cacería de animales, los cuales por este fruto se encuentran más gordos. Por lo anterior, tumbar chonta representa terminar con una de las fuentes principales de alimentación, generando a su vez la necesidad de una movilidad hacia un nuevo territorio. Así, tiene una representación compleja que significa terminar con la fuente de subsistencia y buscar un nuevo territorio de reproducción, en un ejercicio marcado por la tradición y que exige buscar nuevos referentes de antepasados. De la acción de tumbar las chontas se procede a seleccionar los segmentos de la palma con la que se confeccionará las lanzas, posterior se trasladan esos segmentos de chonta al onko, donde los hombres darán la forma y los detalles (Narváez Collaguazo 2013c, 2013a, 2016). 
Existe una destreza de los waorani para la confección de las lanzas que se manifiesta en que estas llegan a tener el mismo largo e incluso peso, que evidencia una práctica continua en la elaboración de estos implementos y una gran habilidad. ${ }^{10}$ La confección se realiza fuera del onko, en un espacio que permita la utilización de fuego para "hacer fuerte a la lanza", ${ }^{11}$ e impedir que esta se tuerza. Los detalles de la lanza consisten en un mayor volumen en la punta donde se puedan tallar dientes aserrados que impidan la extracción de la lanza una vez esta atraviese un cuerpo. El número de dientes depende de cada individuo, pero en general tienen al menos cinco filas. La punta tiene como característica tener una forma cónica romboidal en cuya base se tallan los dientes, mientras el lomo de la punta es redondeado como para permitir una mayor penetración en el cuerpo. El segmento medio de la lanza es tallado con un menor diámetro, de manera a permitir que una persona pueda portar varias lanzas en cada mano.

Las lanzas se dejan secar, apoyadas para que no se tuerzan. Mientras las lanzas se secan los hombres van preparando las plumas que utilizarán para la decoración, las fibras y otros elementos, que tanto por su color o por su origen determinarán las características de la lanza. ${ }^{12}$ La lanza lleva decoración en sus tres segmentos: cerca de la punta, en el lugar donde se empuña y se porta la lanza, y en el segmento final. Tanto en el sector de la punta como en el final la decoración utilizada son fibras de chambira, plumas y cerdas de animales. De esta manera las lanzas resumen la complejidad del pensamiento de la sociedad waorani en torno a su visión del mundo y sobre todo en torno a la acción de la muerte, además del contexto y momento histórico en el que se encuentra.

\section{EL CICLO RITUAL DE LA GUERRA}

Sobre la base de todo este contexto histórico e información etnográfica encontramos una relación simbólica entre el concepto de muerte o asesinato en el ethos tribal waorani con la venganza, si bien en wao tiriro no existe un término

10 Los peritajes antropológicos de "Lanzas que ocasionaron la muerte de la familia Duche" (Narvaéz Collaguazo 2010), Peritaje Cultural de las Lanzas que Intervinieron en la Muerte de Mariano Castellanos (Narváez Collaguazo 2010), Alcance al Estudio de Antropología Jurídica (Narváez Collaguazo 2013a) y Análisis Comparativo de Lanzas Tagaeri Taromenane que han Intervenido en Muertes y Ataques (Narváez Collaguazo 2013b) identifican patrones recurrentes en lo que se refiere al largo y peso de las lanzas, así como en la decoración. Además, establecen diferencias entre lanzas con lo que se concluyó que estas fueron confeccionadas por diferentes personas. De igual manera se pudo identificar, a partir de las características antes indicadas, que los individuos que realizaron el ataque fueron varios y atacaron a más de una de las víctimas.

11 Entrevista a Tehuane Omehuay, junio 2013.

12 Cada guerrero guarda plumas y elementos para decoración de lanzas, y los mantiene hasta que necesita usarlos en decoración. 
que enuncie la venganza como tal (Boster, Yost e Peeke 2003), ${ }^{13}$ la expresión de ira o pinte es la base que da sentido y razón a un ataque o al inicio de la guerra. Ahora bien, la venganza como una acción que busca una reparación a la muerte de un familiar o integrarse a un grupo aliado para una correría de guerra tiene un marco social, y es que la venganza tiene como consecuencia el reafirmar alianzas, principalmente las logradas mediante matrimonio, en donde se integran familiares del o de los emparentados, armando así un grupo amplio de guerreros en alianza que buscarán ejercer una acción que garantice la supervivencia y seguridad del o de los nanicabos.

En este punto, al retornar al análisis de la autarquía es necesario, más aún en un medio selvático en donde existen unas condiciones de igualdad y medios de los nanicabo para obtenerlo del ambiente circundante y apropiarse del mismo, estamos hablando de la recolección que se da en un territorio y que establece el imaginario waorani de territorialidad en el mismo. De esta manera, territorialidad expresa el espacio donde se manifiestan el conjunto de relaciones que un colectivo humano establece con el entorno, a partir de su cosmovisión y forma de reproducción particular (Surrallés 2009). Así, esta condición igualitaria de acceso a un territorio y a unos recursos permite una autonomía de cada nanicabo, dando lugar a varios grupos familiares más sus alianzas, en un espacio de interdependencia e interrelación. En estos espacios el orden es establecido por el código guerrero, que impone la defensa del territorio, y que expresa como fin esa lucha por los recursos de ese territorio, la sobrevivencia y mantener un dominio y control, y por tanto ejercer un poder sobre el mismo a través de la guerra (Clastres 1981; Foucault 2016).

En las acciones de guerra cada grupo busca establecer control sobre el territorio, es decir ejercer el poder, reelaborando las dinámicas relacionadas al conflicto, en donde se reafirman los referentes míticos de la guerra, remitiéndose al derecho dado por los ancestros al territorio o a la tradición de guerra que construye y retoma del ethos tribal waorani que no se ha extinguido, sino que ha sido resignificado en varias formas de códigos guerreros.

Así, la memoria waorani guarda los aspectos vinculados con la cosmovisión y su ethos tribal, en donde el territorio, la defensa del mismo, la guerra, venganza y la muerte con lanzas son parte de su visión de orden" y su concepción de mundo. Así mismo, las dinámicas de movilidad se encuentran vinculadas a esta concepción, en donde destaca la figura materna como línea a la cual se retorna en periodos o ciclos, para hacer uso de los recursos dejados por los abuelos.

13 El estudio de Boster, Yost y Peeke (2003) establece que no existe el término venganza en wao tiriro a fin de no establecer una responsabilidad u obligación de este acto en el grupo familiar atacado, de manera de no generar un contexto de riesgo que podría implicar la venganza. 


\section{BIBLIOGRAFÍA}

BECKERMAN, Stephen, Pamela ERICKSON, James YOST, Jhanira REGALADO, Lilia JARAMILlO, Corey SPARKS, Moises IROMENGA, y Kathryn LONG, 2009, "Life histories, blood revenge, and reproductive success among the Waorani of Ecuador", Proceedings of the National Academy of Sciences, 106 (20): 8134-8139. Disponible en < https://doi. org/10.1073/pnas.0901431106> (última consultación en octubre 2021).

BLOMBERG, Rolf, 2015, Los Aucas Desnudos: Una Reseña de los Indios del Ecuador. Quito: Abya Yala.

BOSTER, James, James YOST, y Catherine PEEKE, 2003, "Rage, revenge and religion: honest signaling of aggression and nonaggression in Waorani coalitional violence", Ethos, 31 (4): 47 1-494. Disponible en < https://doi.org/10.1525/eth.2003.31.4.471 > (última consultación en octubre 2021).

CABODEVILla, Miguel, 1996, Coca: La Región y Sus Historias. Quito: CICAME.

CABOdevilla, Miguel, 1999, Los Huarani en la Historia de los Pueblos del Oriente. Quito: CICAME.

CABODEVILlA, Miguel, 2010, La Nación Waorani. Quito: CICAME, Fundación Alejandro Labaka.

CHAGNON, Napoleon, 1968, Yanomamö: The Fierce People. Nueva York: Holt, Rinehart and Winston.

CLASTRES, Pierre, 1981, "La cuestión del poder en las sociedades primitivas", in Pierre Clastres (ed.), Investigaciones en Antropología Política, 109-1 16. México, DF: FCE.

CLASTReS, Pierre, 2004, Arqueología de la Violencia: La Guerra en las Sociedades Primitivas. México, DF: Fondo de Cultura Económica.

CLASTRES, Pierre, 2008, La Sociedad contra el Estado. Madrid: Terramar.

FOUCAUlT, Michel, 2016, La Sociedad Punitiva. México, DF: Fondo de Cultura Económica. GARTelmanN, Karl Dieter, 1977, El Mundo Perdido de los Aucas. Quito: S.E.

GOLDMAN, Marcio, 2003, "Os tambores dos mortos e os tambores dos vivos. Etnografia, antropologia e política em Ilhéus, Bahia”, Revista de Antropología, 46 (2): 445-476. Consultado en < https://doi.org/10.1590/S0034-77012003000200012 > (última consultación en octubre 2021, ya no disponible).

MA OMENE, Manuela, 2012, “Saberes waorani y Parque Nacional Yasuní: plantas, salud y bienestar en la Amazonía del Ecuador”, Iniciativa Yasuní ITT, Ministerio Coordinador de Patrimonio, Ministerio del Ambiente, Programa de Naciones Unidas para el Desarrollo, y Fondo para el Medio Ambiente. Quito: S.E.

MACFARLAN, Shane, Pamela ERICKSON, Jost YOST, Stephen BECKERMAN, Jhanira REGALADO, y Lilia JARAMILLO, 2018, "Bands of brothers and in-laws: Waorani warfare, marriage and alliance formation". Proceedings of the Royal Society B: Biological Sciences, 285 (1890). Disponible en < https://doi.org/10.1098/rspb.2018.1859 > (última consultación en octubre 2021). Maldonado, Pedro, 1750, Carta de la Provincia de Quito y de las Adyacentes, Obra Posthuma. Hecha sobre las observaciones astronómicas y geográficas de los Academicos Reales de las Ciencias de Paris y de las Guardias Mar de Cadiz y tambien de los R.R.P.P. Misioneros de Maynas [Map]. Disponible en < https://exhibits.stanford.edu/ruderman/catalog/fz677ws9017 > (última consultación en octubre 2021).

MAUSS, Marcel, 2010, Ensayo sobre el Don: Formas y Funciones del Intercambio en las Sociedades Arcaicas. Buenos Aires: Katz Editores. 
MORENO YÁNEZ, Segundo, 2018, Simbolismo y Ritual en las Sublevaciones Indígenas. Quito: Universidad Andina Simón Bolívar y Corporación Editora Nacional.

NARVÁEZ COllaguazo, Roberto, 2010, Peritaje Cultural de las Lanzas que Intervinieron en la Muerte de Mariano Castellanos [Informe de Pericia de antropología cultural]. Quito: Fiscalía de Orellana.

NARVÁEZ COLLAGUAZO, Roberto, 2013a, Alcance al Estudio de Antropología Jurídica [Informe de Pericia de antropología cultural]. Quito: Comisión de la Verdad y Derechos Humanos de Fiscalía General del Estado.

NARVÁEZ COLlaguAZO, Roberto, 2013b, Análisis Comparativo de Lanzas Tagaeri Taromenane Que Han Intervenido en Muertes y Ataques [Informe de Pericia de antropología cultural]. Quito: Comisión de la Verdad y Derechos Humanos de Fiscalía General del Estado.

NARVÁEZ COLLAGUAZO, Roberto, 2013c, Estudio de Antropología Jurídica sobre los Hechos Ocurridos en el Yasuní, en el Poblado de Yarentaro [Informe de Pericia de antropología cultural]. Quito: Comisión de la Verdad y Derechos Humanos de Fiscalía General del Estado.

NARVÁEZ COLLAGUAZO, Roberto, 2016, "Intercambio, guerra y venganza: el lanceamiento de Ompure Omehuai y su esposa Buganei Caiga”, Antropología Cuadernos de Investigación, 16: 99-110.

NARVÁEZ COLLAGUAZO, Roberto, 2018a, "La etnografía: instrumento de investigación en antropología jurídica. El caso de un pueblo amazónico”, Revista Temas Sociológicos, 23: 307-341.

NARVÁEZ COLLAGUAZO, Roberto, 2018b, “Territorialidad de los grupos familiares de pueblos indígenas en aislamiento (PIA) en la Región del Yasuní, Amazonía Ecuatoriana", Tipití: Journal of the Society for the Anthropology of Lowland South America, 16 (1): 103-119.

NARVÁEZ COLlaGUAZO, Roberto, y Patricio TRUjILlo mONTALVO, 2020, “Tiempos de guerra y tiempos de paz, continuum simbólico de un pueblo de reciente contacto: el caso etnográfico de los waorani en la Amazonía ecuatoriana", Cadernos de Campo, 29 (1): 38-52.

OLIVEIRA, Roberto Cardoso de, 1996, “La antropología latinoamericana y la 'crisis' de los modelos explicativos: paradigmas y perspectivas”, Maguaré, 11-12: 9-23.

RIVAL, Laura, 1996, Hijos del Sol, Padres del Jaguar. Quito: Abya Yala.

RIVAL, Laura, 2015, Transformaciones Huaoranis: Frontera, Cultura y Tensión. Quito: Universidad Andina Simón Bolívar, Latin American Centre, University of Oxford: Abya Yala.

RIVAS TOLEDO, Alex, y Rommel LARA PONCE, 2001, Conservación y Petróleo en la Amazonía Ecuatoriana: El Caso Huaorani. Quito: Abya Yala.

ROBARCHEK, Carole, y Clayton ROBARCHEK, 1998, Waorani: The Contexts of Violence and War. Nueva York: Fort Word.

SANTOS ORTIZ, Juan, 1996, Los Últimos Huaorani. Quito: CICAME.

SOPRANO, Germán, 2008, "Doña Silvia: análisis de redes políticas en el peronismo de la provincia de misiones durante una campaña electoral municipal”, Andes, 19: 119-155.

SURRALLÉS, Alexandre, 2009, "Entre derecho y realidad: antropología y territorios indígenas amazónicos en un futuro próximo", Bulletin de l'Institut Français d'Etudes Andines, 38 (1): 29-45. Disponible en < https://doi.org/10.4000/bifea.2789 > (última consultación en octubre 2021).

TIRDMARSH, Wilfred,1945, Unarranged Notes on the Aucas of the Nushiño Basin. Mimeografiado. 
TOCA, Aca, Omari IMA, Cawo BOYA, Tiyane IMA, Wari PAA, Gomoque OMENE, Wanuka TOCARE, Mencay IMA, Naware ENKERI, Tepaa BAIHUA, y Ariadna REYES, 2009, Tededanipa: Las Voces de las Mujeres. Quito: Corporación Humanas, AMWAE, Ministerio de Cultura del Ecuador.

TRUjIllo mONTAlvo, Patricio, 2001, Salvajes, Civilizados y Civilizadores: La Amazonía Ecuatoriana, el Espacio de las Ilusiones. Quito: Abya Yala y Fundación de Investigaciones Andino Amazónicas.

Trujillo montalvo, Patricio, 201 1, Boto Waorani, Bito Cowuri: La Fascinante Historia de los Wao. Quito: FIAAM.

TRUJILlO MONTALVO, Patricio, 2016, "Código guerrero: movilidad, guerra y muerte con lanzas”, Antropología Cuadernos de Investigación, 16: 85-98.

TRUJILlO MONTALVO, Patricio, 2018, "Identificación y dinámicas de los Pueblos Indígenas en Aislamiento Voluntario (PIAV) en el Yasuní”, Boletín de Antropología, 33: 271-296.

TRUjILlo, Jorge, 2019, El Otro en la Historia: Los Waorani antes del Contacto. Quito: Fundación Labaka, FEPP, Unión Europea.

UP DE GRAFF, Fritz, 1996, Cazadores de Cabezas del Amazonas. Quito: Abya Yala.

WALLIS, Emily, 1973, Aucas Downriver. Nueva York: Harper \& Row.

YOST, James, 1981, "Twenty years of contact: the mechanisms of change in Wao (Auca)", in Norman Whitten (ed.), Cultural Transformations and Ethnicity in Modern Ecuador. Urbana, IL: University of Illinois Press, 667-704.

YOST, James, 1989, "Veinte años de contacto: los mecanismos de cambio en la cultura huao", Amazonía Ecuatoriana: La Otra Cara del Progreso. Quito: Abya Yala, 261-286. 